\title{
Laser-emission microscopy
}

An optical setup for observing targeted fluorophores lasing from within tissue.

Cancer biomarkers can help researchers identify diseased tissues. Such biomarkers include the abundance of nucleic acids or the amount of particular proteins in a cell or tissue. Biomarkers are typically imaged in tissue slices with light and fluorescence microscopy. However, histology, immunohistochemistry, and immunofluorescence approaches are sometimes difficult to interpret because of issues such as low contrast and signal to background.

Xudong Fan at the University of Michigan and colleagues developed an alternative, complementary microscope for imaging cancer biomarkers. Their system turns targeted fluorescent dyes into 'biolasers', for which the laser emission (rather than fluorescence) is recorded. Compared with fluorescence imaging, imaging laser emission within samples has advantages, includ- ing narrow emission spectra, high intensity, and high sensitivity to cellular environment.

A laser typically consists of three components: a pump source, a gain medium, and an optical resonator. The pump source illuminates the sample and excites photons in the gain medium, which is the fluorescent dye in this case, and the optical resonator traps the photons, which causes them to amplify and ultimately results in laser emission.

In Fan's laser-emission-based microscope (LEM), tissue slices that are labeled using fluorophore-conjugated antibodies toward specific proteins or nucleic acid dyes are sandwiched between two dielectric mirrors that form a Fabry-Pérot cavity (the optical resonator). This sample holder is then integrated with a $2 \mathrm{D}$ raster-scanning stage for imaging. Although not the first demonstration of intracellular lasing, the researchers note that this is, to their knowledge, the first use of intracellular lasing to image biomark- ers within tissues from human patients.

The researchers examined tissue samples from patients with lung and colon cancer. They imaged labeled nucleic acids or protein biomarkers at a resolution of less than $700 \mathrm{~nm}$. Their method was used to distinguish between cancer and healthy tissue, and was even able to recognize early-stage lung cancer. Cancer cells and healthy cells could be distinguished based on the abundance of individual biomarkers, as the differences in the amount of fluorophore present lead to large differences in lasing thresholds. This work represents an important step forward for cancer imaging and imaging of lasing from within tissues.

\section{Rita Strack}

\section{RESEARCH PAPERS}

Chen, Y.-C. et al. Laser-emission imaging of nuclear biomarkers for high-contrast cancer screening and immunodiagnosis. Nat. Biomed. Eng. 1, 724-735 (2017). 